\title{
EN TORNO AL DEBILITAMIENTO DE UN ROL PROFESIONAL
}

ON THE WEAKENING OF A PROFESSIONAL ROLE

Mónica Llaña Mena
Magister en Educación
Universidad de Chile
mllana@uchile.cl

Resumen: El presente artículo tiene como propósito analizar la situación de deslegitimación del rol docente. Se postula su carácter técnico en detrimento de su rol pedagógico en la realidad institucional actual. Como sustentación, se entregan antecedentes a nivel de los cambios que han experimentado la sociedad chilena y su impacto en el sistema educativo nacional. Cambios culturales a nivel profundo en los que las nuevas tecnologías penetran en los espacios escolares transformando la vida cotidiana en ellas y generando heterogéneas demandas pedagógicas, y relacionales. Se describen además las tensiones y desafíos que implican para una acción profesional, el ser coartada por una racionalidad tecnológica que valora y potencia en la escolaridad, la eficiencia, eficacia y productividad. El profesor, parte el mundo de la vida, enfrenta complejos procesos de individuación en los que debe responsabilizarse de manera personal del desempeño de su rol. Ante un sistema controlador y coercitivo, que ha limitado su autonomía, su participación en decisiones pedagógicas, se incuba una fuerte tensión con el mundo de la vida, de la intersubjetividad compartida en la escuela. El profesional en consecuencia experimente un fuerte debilitamiento de su rol profesional.

Palabras claves: deslegitimación, rol profesional, contexto sociocultural, individuación, mundo de la vida, sistema.

Abstract: The purpose of this article is to analyze the situation of delegitimization of the teaching role. Its technical character is postulated to the detriment of its pedagogical role in the current institutional reality. As support, background information is provided on the changes experienced by Chilean society and their impact on the national education system. Cultural changes at a deep level in which new technologies penetrate school spaces, transforming daily life in them and generating heterogeneous pedagogical and relational demands. It also describes the tensions and challenges that imply for a professional action, being constrained by a technological rationality that values and promotes efficiency, efficacy and productivity in schooling. The teacher, part of the life-world, faces complex processes of individuation in which he/she must take personal responsibility for the performance of his/her role. Faced with a controlling and coercive system, which has limited their autonomy, their participation in pedagogical decisions, a strong tension is created with the life-world, of the intersubjectivity shared in the school. Consequently, the professional experiences a strong weakening of his professional role. 
Key words: delegitimization, professional role, sociocultural context, individuation, lifeworld, system.

\section{INTRODUCCIÓN}

Inéditas concepciones están rediseñando la realidad del sistema educativo y frente a las cuales profesores y profesoras no son reconocidos como actores estratégicos, convirtiéndose en parte de un entramado que contiene disímiles e impredecibles transformaciones a nivel de sociedad global.

En ese marco, el profesorado recoge las exigencias de un racionalismo que, convertido en tendencia dominante, concibe la calidad humana como sinónimo de eficacia y eficiencia. Es preocupante constatar el predominio de las lógicas estandarizadas en la educación formal que excluyen a quienes no cumplen con los estándares prescritos, agudizando así la perpetuación de las desigualdades en nuestra sociedad.

Son radicalmente nuevos los problemas que enfrentan estos profesionales, a punto de poner en crisis su tradicional identidad profesional. Su desempeño pedagógico, además de resistir la vorágine de los cambios culturales, los obliga a asumir responsabilidades de tipo administrativo, manejar adecuadamente el escaso tiempo, comprometerse a lograr metas y objetivos, muchos de ellos a corto plazo.

Las altas exigencias a la labor docente producen una disociación entre ellas y una serie de dispositivos que constriñen, regulan y norma la acción profesional. Manda la consecución de resultados efectivos para un sistema mediante la evaluación de logros estandarizados. Hay una pérdida de autonomía que puede provocar efectos en plazos no previsibles, porque ya se estarían generando cuestionamientos respecto a su saber, estigmatizado por una opinión socialmente generalizada de una formación precaria.

En el actual contexto histórico y en la encrucijada marcada por fuertes presiones en las que prima un control autoritario de la acción pedagógica, no es aventurado inferir que, como profesionales, están siendo conducidos a asumir un rol más técnico que profesional.

Creemos importante aventurarnos a analizar el tránsito desde los cambios contextuales a las racionalidades dominantes en el espacio educativo y que están incidiendo en estas transformaciones.

\section{EL SISTEMA EDUCATIVO EN EL CRUCE DE UNA ÉPOCA DE PROFUNDAS TRANSFORMACIONES SOCIOCULTURALES}

La educación en nuestro país lleva la marca de una nueva época en la cual y desde la perspectiva de la formación de las nuevas generaciones, se vuelve imperioso desafío el acceder a la comprensión de la acción profesional de quienes se encuentran en la incertidumbre de haber sido expulsados de antiguas matrices pedagógicas, actualmente difuminadas. Una pérdida de magnitud que ha incidido en el tránsito de un sujeto pedagogo a un profesor técnico. 
Enfrentamos un escenario de transformaciones históricas multidimensionales que afectan al mundo en general, y que, en Chile, han tenido un impacto específico en los sistemas productivos, organizativos, culturales e institucionales, en un ámbito de profundos cambios tecnológicos.

Vivimos un nuevo período histórico, una realidad de cambios abruptos, innovaciones y redes en el que las grandes empresas financieras y mediáticas, se imponen sobre las instituciones tradicionales, familia, escuela (Touraine, 1997).

Para este autor, la reflexión sobre las sociedades contemporáneas se debería sostener en la constatación de la existencia de una creciente disociación entre el mundo de las economías, mundo instrumental y las culturas, mundo simbólico que convive con la aceleración de los cambios, circulación de capitales, e informaciones instantáneas.

Es inquietante constatar las insospechadas aristas que esta realidad tiene sobre los fines de la educación formal y por ende sobre un rol clave como es el de un profesor formador.

Por otra parte, la expansión del mundo tecnológico y su enorme capacidad de difusión se introduce en el sistema educativo desde múltiples planos y niveles.

Los efectos del poder de penetración de las nuevas tecnologías, su capacidad de transmisión de la información, y de interconexión intervienen en todas las actividades humanas.

Culturalmente, las proyecciones de la información instantánea desafían, al conocimiento, desvalorizado frente a una información abrumadora, producen cambios en las relaciones interpersonales, en los códigos comunicativos, valóricos, éticos, todo en una estructura holísticamente diseñada a través de una morfología de redes.

Surge y se impone un nuevo paradigma, que, asociado a las tecnologías de la información, posee por una lógica reticular que determina su influencia en una amplia gama de procesos y organizaciones. Su adaptación a la complejidad de las interacciones amplía sus posibilidades creativas en múltiples interacciones (Castells, 1999).

En estos marcos de movimientos y aceleraciones, se generan por otra parte importantes contradicciones que se vuelcan en la escolaridad, en especial las nuevas tecnologías, que circulan, determinan, controlan y dirigen la vida cotidiana en las escuelas. Se sitúan desde una racionalidad basada en la eficacia, eficiencia y productividad, imbricadas en las crecientes demandas de mayor igualdad y participación en la vida social (Ritzer, 2002, Bauman, 2006).

Es preciso subrayar, además, que el ethos cultural de la época muestra una valoración exagerada del sujeto, un narcicismo que devela un individuo emancipado de cualquier marco trascendental (Lipovetsky, 2000).

Para este autor, este fenómeno se traduce en una mutación antropológica, un nuevo estado del individualismo.

Un individualismo en que los medios, la televisión, en las redes sociales ocupan un espacio importante en la vida del hombre contemporáneo, ya que le permiten empaparse de 
información y acontecimientos acaecidos a nivel mundial, y que en el ámbito de su vida privada devienen un apoyo en la búsqueda de experiencias de fácil satisfacción (Touraine, 1997).

Muchas de esas expectativas están orientadas por el consumo de masas que ha generado valores igualitarios y una demanda de mayor horizontalidad en las relaciones humanas. (Araujo y Martuccelli, 2012).

Es posible inferir, por consiguiente, que las proyecciones de estos cambios en la configuración del rol profesional son radicales y que un solo aspecto, a manera de ejemplo, la disciplina, es desvalorizada, siendo reemplazada por exigencias de satisfacción inmediata. El esfuerzo pierde importancia. Se ha producido una pérdida de la autoridad tradicional al interior de las instituciones educativas.

Incuestionablemente, y de acuerdo a lo expuesto, el sistema educativo ha sido profundamente afectado por una realidad que lo impacta en diferentes planos y niveles. Culturalmente porque se han creado nuevos códigos, lenguajes, formas de interactuar, y una multiplicidad de formas culturales, que impregnan la vida escolar. Pedagógicamente, porque emerge la urgente necesidad de crear nuevas estrategias didácticas, nuevos vínculos en las relaciones educativas, un currículo pertinente a las diferencias y heterogeneidades entre otras complejidades.

Uno de los aspectos centrales de este enjambre de nuevas realidades, ha sido la globalización y la virtualización de las relaciones sociales, que han potenciado las interrelaciones entre personas que se haya en lugares distantes, y que pueden comunicarse y establecer relaciones interpersonales en tiempo real (Giddens, 1996).

Situación que ha producido un profundo impacto en, a lo menos, tres aspectos fundamentales a considerar desde la perspectiva del rol profesional. En la construcción de las identidades a través de las cuales las personas constituyen su sentido de vida, los proyectos que impulsan los distintos actores sociales a partir de sus valores e intereses compartidos y las formas de comunicarse, por lo tanto, de aprehender, y de relacionarse entre las personas. Es imposible soslayar el hecho que estos ejes constituyen un importante desafío para la actualización del rol docente.

\section{UN PROFESIONAL INTERPELADO}

La comprensión e interpretación del rol profesional, no puede escindirse de las transformaciones históricas ni de las fracturas que subyacen en la estructura del sistema educativo. Tanto las reformas y las políticas públicas, la carrera docente, los sistemas de evaluación han constituido procesos que fueron moldeando las experiencias y las prácticas pedagógicas. Si nos remontamos en el tiempo, en la década de los 80, la municipalización, marcó un hito relevante que aun incide significativamente, no solo en la gestión sino también en los propósitos de equidad y calidad.

El profesor, sumergido en ese espacio, la escuela, actúa y acumula experiencias en las que políticas públicas, se encarnan en prescripciones que dominan los procesos educativos. 
Se construye como individuo en el ámbito profesional desde las estructuras y racionalidades de esas políticas.

Como sujeto histórico, es exigido e interpelado por una sociedad en ritmos y polaridades, diferenciadas por el cambio como categoría central, experimenta y vive un complejo proceso de individuación.

Proceso que implica un doble juego, imbricado desde las experiencias personales sedimentadas, a la articulación de la realidad del sistema educativo en su contexto, configurando su constitución como individuo profesor (Martuccelli, 2012).

La individuación, y la demanda de tomar decisiones personales en sus acciones de vida, no significan la eliminación de desigualdades y/o exclusiones sociales o que todas las personas se encuentren en las mismas condiciones para elegir su vida. Lo que ocurre es que como las desigualdades son atribuidas a las personas, pertenecen al ámbito privado. Complejas exigencias para ejercer un rol profesional. En su carácter de imperativo social, la individuación, induce a los individuos a hacerse cargo de sí mismos, desde sus diferentes y desiguales posicionamientos, lo que constituye e impulsa una forma de privatización de la vida social.

¡Difíciles procesos para abordar procesos formativos en la actual conformación de la sociedad!

El proceso de individuación se vincula con los procesos de reflexividad es decir con una racionalidad que es capaz de pensar y decidir sobre todos los asuntos públicos y privados de manera crítica (Ritzer, 2002).

Racionalidad que incide en los tensionados estilos de comunicación, y heterogéneas prácticas en las comunidades educativas, lo que constituye muchas veces una fuente de ruptura para la legitimidad de un rol profesional, que se enfrenta en la cotidianeidad del espacio escolar a múltiples racionalidades en las que subyacen diferentes universos de sentido respecto a la escolaridad.

Es importante reconocer la realidad de un fenómeno recurrente en las sociedades contemporáneas, que genera una la producción permanente de saberes sobre la vida social, puesto que las informaciones cambian, se complementan, se niegan unas a otras, a gran velocidad y lo que fue verdad indiscutida en un momento, puede ser fácilmente refutado, lo que incide en la relativización de las nociones, conceptos y valoraciones absolutas sobre las ideas y juicios. En contextos de modernidad reflexiva, el individuo está a cargo de decidir sobre las cosas, ya que las instituciones pierden su rol en la estandarización de estas decisiones. Ámbito de decisiones que colocan en una encrucijada al profesor quien debe intentar posicionarse desde una legitimidad cuestionada por esta eclosión de saberes naturalizados desde el conocimiento común.

La razón es que la reflexividad permea variados ámbitos de la vida social y de las relaciones sociales y puede observarse en la relación entre individuos y grandes instituciones como en las relaciones en la escuela, en la familia, en comunidades y territorios, entre profesores y estudiantes, padres e hijos, amigos. Nutre toda la vida cotidiana en un determinado espacio social. 
Desde estas nuevas realidades, emergen nuevas problemáticas que instalan nuevamente el desafío de pensar acerca del sentido de la educación en Chile. Así como también la urgencia de legitimar, reformular el rol del profesor, demandado por un nuevo contexto epocal y asumiendo las contradicciones de las transformaciones sociales.

\section{EL MUNDO DE LA VIDA EN LA ESCUELA, UN ESCENARIO ATRAPADO POR EL SISTEMA}

Dada la profundidad de las transformaciones descritas en los párrafos precedentes, no es posible abordar todos los ámbitos que se verán impactados por éstas y que tienen incidencia en la configuración del rol docente en el actual período histórico.

El análisis a desarrollar se sustenta en una postura que supera la oposición entre objetivismo y subjetivismo, entre sujeto y objeto y que limita la imbricación individuo sociedad.

Es interesante destacar, para contribuir a develar algunos mecanismos que subyacen a la situación de desprofesionalización del profesorado en nuestro país, el aporte de dos concepciones asociadas a la comprensión de la sociedad desde la perspectiva de los sujetos en acción. Nos referimos a las posturas teóricas de Jurgen Habermas (1987) y Alfred Schutz (1973) que intentaremos conectar para ampliar la comprensión de las tensiones que experimenta el rol docente.

Para el primero de ellos hay una sola sociedad, el mundo de la vida y el sistema, solo hay maneras diferentes de considerarlos.

Desde la perspectiva analítica sustentada por Habermas, los actores sociales, profesores, son miembros de la sociedad que es el mundo de la vida y cuyas relaciones con ese mundo se enmarcan en el ámbito de algo que tiene lugar en el mundo objetivo, reconocido, compartido y también parte de algo al que los actores otorgan significado. Es el mundo de la experiencia común, estructuralmente conectado a la cultura y al lenguaje. Al estar constituido según el autor por tres mundos: objetivo, social y subjetivo, es importante destacar que en el plano de la acción pedagógica los tres constituyen un marco de interpretación a partir del cual se elaboran definiciones comunes de una situación. A manera de ejemplo, si un directivo, jefe de la Unidad Técnico Pedagógica, ordena la realización en un tiempo acotado, de una programación actualizada de la docencia, la jerarquía formal es el marco normativo. El mandato constituye una orden que se deberá cumplir, sin sobrepasar el horizonte en que este se sitúa, el mundo de la vida.

Para Habermas, el mundo de la vida representa el punto de vista de los sujetos, en cambio el sistema, que también tiene sus raíces en el mundo de la vida, representa una percepción externa y desarrolla sus propias características estructurales, estado, economía, familia. Estas estructuras evolucionan y se diferencian cada vez más del mundo de la vida, aumenta su autosuficiencia y su poder, capacidad de controlar e imponerse al mundo de la vida. En el sistema educativo, esta diferenciación se observa en la deslegitimación y desvalorización de las heterogeneidades culturales, encarnadas en diferentes códigos simbólicos, tradiciones y rituales que conforman disímiles ethos culturales, y que han sido invisibilizados en la formación de las nuevas generaciones. 
En el mundo moderno, el sistema controla cada vez más el mundo de la vida, observándose un creciente poder sobre ésta, y desde su creciente complejidad, agota la capacidad del mundo de la vida que tiende a instrumentalizarse.

Como explicita Habermas:

La pauta capitalista de la modernización está marcada por una deformación, una reificación de las estructuras simbólicas del mundo de la vida bajo los imperativos de los subsistemas que se diferencian a partir del dinero y el poder que se convierte en autosuficiencia (Habermas, 1987, p.155).

Como no conectar esta cita con la realidad de una educación convertida en mercancía, sometida al mercado, a un poder controlador de la gestión pedagógica, y cuando esa racionalidad, la del sistema se impone sobre la racionalidad del mundo de la vida, colonizándola.

Complementariamente desde la perspectiva fenomenológica de Schutz y Luckmann el mundo de la vida debe comprenderse para actuar sobre él, desde la actitud natural lo que presupone una actitud del sentido común. Es un mundo que se da por establecido, como realidad que se presenta como mundo intersubjetivo compartido por otros.

La escuela es parte del mundo de la vida cotidiana y los miembros de esa comunidad comparten un ámbito de significaciones e interpretaciones a partir de una realidad que se da por sentada, parte de un mundo intersubjetivo compartido por todos quienes son parte de ella.

Quienes participan de la vida cotidiana en ese espacio, interactúan permanentemente, en las aulas, los patios, en las salas de profesores, en las oficinas, bibliotecas, laboratorios, gimnasios, generando rutinas, rituales, comunicaciones que conforman una estructura simbólica e intersubjetiva.

A manera de ejemplo, un profesor situado en el espacio escolar, que es un espacio social, la escuela, es parte a su vez de un horizonte móvil que se ubica desde el entorno, los demás profesores y directivos, los estudiantes, sus familias, todos partícipes de la complejidad de ese mundo de la vida, sin que él, como sujeto, pueda marginarse de ese horizonte fluido y complejo, pero interpretado desde la actitud natural.

Habría que precisar además que las relaciones interpersonales, son partes constituyentes de la realidad del mundo de la vida cotidiana (Schutz y Luckmann, 2002). Realidad que parece ser evidente para quienes son parte de ella. Desde la perspectiva de los profesores, es el mundo incuestionado, producto de su experiencia, allí se ubican las situaciones a resolver. Ese mundo cotidiano, alojado en la escuela, pasa ser el escenario que pone límites a la propia acción profesional y a la acción con los demás miembros de esa comunidad.

Sin desconocer que la escuela es parte de un mundo social históricamente producido que constituye para los actores un marco de referencia, parte de un mundo natural, 
interpretado como tal en que los integrantes, pero también sobre el cual pueden actuar como sujetos.

Cabe destacar, en especial en relación a las tensiones que estarían deslegitimando ese rol profesional, que, en el marco de la complejidad de los nuevos contextos, que penetran y tensionan las instituciones educativas, surgen problemáticas que sobrepasan su acervo de conocimiento ya que no corresponderían a los marcos de referencia asumidos como válidos ni coherentes con la experiencia acumulada. Sería importante preguntarse si el profesorado estaría en condiciones de enfrentar situaciones problemáticas en una realidad compleja como la que vive desde su experiencia cotidiana.

Un profesional que, compartiendo la vida cotidiana en la escuela, configurado como campo educativo y desde la concepción Bourdeniana de espacio de fuerzas, es parte de un mundo práctico en el que busca, elige formas de actuar.

En ese plano, se puede hablar de los condicionamientos asociados a formas de vida laboral, lo que implicaría hacer relevante el situar al profesorado como poseedor de un habitus pedagógico que de acuerdo a Bourdieu corresponde a un "sistema de disposiciones duraderas y transferibles, estructuras predispuestas a funcionar como estructuras estructurantes, como principios generadores y organizadores de prácticas y representaciones" (Bourdieu, 1997, p. 92).

Con los habitus se constituye el mundo práctico como sistema de disposiciones y motivaciones, un mundo de fines ya realizados, orientador de modos de empleo o caminos a seguir, que tienden a aparecer como naturales ya que son parte de los principios de percepción y adaptación incorporados.

Habría una correlación muy estrecha entre probabilidades objetivas científicamente construidas, en el espacio profesional, como las referidas a la carrera docente, a sus sistemas de evaluación, por ejemplo y las esperanzas subjetivas de ese cuerpo profesional. Y entonces es muy probable que las prácticas del profesorado, relacionadas con ascensos, remuneraciones, jornadas laborales y un largo etcétera, se encuentren para ellos en el ámbito de lo irrealizable e impensable a corto plazo.

El habitus, es preciso reconocer, permite la producción libre de pensamientos, percepciones y acciones inscritas dentro de los límites que marcan las condiciones de producción reconociendo la capacidad de generación infinita. Esto significa que, de acuerdo a la realidad del contexto contemporáneo, sería fundamental reconocer la capacidad de generar percepciones, acciones, expresiones en el marco de las condiciones históricas y sociales. Respecto al habitus pedagógico, sería necesario comprenderlo no solo en relación a las condiciones en que se configuró, a través del período de formación, y del ejercicio profesional, sino que en relación a las condiciones sociales en que se manifiesta y que, de acuerdo a lo señalado, correspondería a profundos cambios en la sociedad.

Como principio generador, el habitus pedagógico reactivaría el sentido objetivado de las instituciones educativas que se reproducen como disposiciones duraderas, condicionando su funcionamiento, su vigencia, aunque imponiéndoles revisiones y cambios. El habitus pedagógico homogeniza, al compartir condiciones de existencia, conlleva el manejo de códigos comunes y las movilizaciones colectivas que para tener éxito deberían 
tener un mínimo de concordancia entre los actores poseedores de habitus similares, como clase o grupo profesional.

Se actualiza en el campo educativo, como red de relaciones entre las posiciones objetivas en su interior, directivos, profesores, estudiantes, comunidad educativa, aunque constreñidos por la estructura de ese campo. Para Bourdieu el campo educativo es un tipo de mercado competitivo en el que se despliegan varios tipos de capital, siendo el político el más importante. Obviamente las posiciones de los agentes al interior del campo dependen de la cantidad y peso relativo del capital que poseen. Es innecesario destacar que, en la actual situación de la escuela, el profesor como agente tiene escaso poder en los procesos educativos formales (Bourdieu y Wacquant, 1992).

En síntesis, los profesores producen sus prácticas, las perciben y evalúan, en medio de una red de relaciones, que posee una lógica específica, en el que se emplean y despliegan varios tipos de capital, económico, cultural, social y simbólico. Esa lógica proyecta una rigidez estructural en todo el sistema educativo.

Dicha rigidez estructural, dificulta la incorporación de los códigos de conducta comprensivos en las comunidades educativas por el contrario sus prácticas cotidianas en el ambiente institucional muestran un clima de tensiones, rencores y frustraciones, sensación de estar estigmatizados, y de no ser sujetos dignos de confianza.

\section{Y EN ESE CONTEXTO ¿PEDAGÓGO O TÉCNICO?}

En el ámbito de estas nuevas complejidades, todo el análisis de las situaciones paradojales que vivencia el profesorado pasa por la necesidad de reconocer como las estructuras y las transformaciones globalizadas en sociedades red (Castells, 1997), modelan la configuración y el ejercicio de un rol profesional. Proponemos develar en un proceso de doble hermenéutica, sostenido por el habitus pedagógico como eje estructural, una práctica imbricada en una urdimbre de experiencias y tensiones.

Las tensiones que configuran una representación compleja del rol profesional se sustentan básicamente en sus condiciones laborales, marcadas por la creciente pérdida de control del trabajo que realizan, deficitarios tiempos y espacios para ejercer su trabajo, predominio de búsqueda de resultados a través de reformas implementadas con criterios del mercado laboral, regulaciones no participativas del trabajo docente entre otras situaciones.

Parte de un sistema escolar, las instituciones educativas se caracterizan por mantener un orden jerárquico y burocrático. Siguiendo a Max Weber (2002) estaríamos en presencia de una racionalidad formal, de medios afines, que esconde una profunda irracionalidad en los marcos de un capitalismo avanzado, un neoliberalismo que propugna una racionalidad centrada en la eficiencia, eficacia, productividad y deshumanización.

Convertida en institución cerrada, se resiste transformar sus estructuras profundas, su ordenamiento jerárquico, su no reconocimiento de las formas autoritarias de ejercicio del poder que circula en su interior, marcando sus espacios de profundas asimetrías. Entonces ¿cuáles serían las contradicciones que encarnan este entramado de circunstancias y que están evidentemente contribuyendo a desprofesionalización de su rol? 
¿No cabría pensar que frente a la exigencia de ser sujeto formador, no encarnaría el pensar técnico un modo de pensar negando todo lo que signifique autonomía en su acción?

Para Heidegger el gran peligro de la técnica moderna es su tendencia totalizadora, ¿no lo son las pruebas estandarizadas? ¿En su pretensión de envolver toda la realidad? Basta recordar las actuales formas de evaluación escolar para compartir el planteamiento de que en ese tipo de actividad se exige como criterio de calidad, la eficacia y eficiencia de los procesos educativos.

Entraña la imposición que se constituye en el peligro de subjetivizar las cosas, llevando al hombre a la deshumanización y la técnica a su auto expansión indefinida lo que implica el establecimiento de una racionalidad tecnológica en todos los ámbitos (Heidegger, 1962).

Se podría entonces inferir que el profesor/técnico estaría amenazado como sujeto por la expansión de la técnica moderna en su racionalidad expansiva, y porque está siendo conducido, por la imposición de la técnica en toda su vida profesional a tecnificarse como sujeto.

Reconociendo que habitamos un mundo tecnificado, habría que admitir el riesgo de que esa racionalidad apague y acalle la conciencia de los individuos. Como reiteran Adorno y Horkheimer tanto la técnica como la tecnología han favorecido la instauración del estado de bienestar, pero han ocultado contradicciones, miserias y peligros de nuestro sistema capitalista y globalizado.

Lo más inquietante en el caso de la profesión docente, es el deterioro de un rol estratégico que lo está convirtiendo en una pieza más de la sociedad tecnificada.

Múltiples deberían ser las opciones para revertir esta realidad. Difícil aventurar respuestas. Tal vez lo más importante sea asegurar una formación que los convierta en intelectuales comprometidos como sujetos activos, históricamente situados, capaces de ejercer poder, comprendido como capacidad de transformación de la acción pedagógica.

\section{REFERENCIAS BIBLIOGRÁFICAS}

Bauman, Z., (2006). Modernidad Líquida. Fondo de Cultura Económica Argentina S.A.

Berger, P. y Luckmann, Th., (2001). La construcción Social de la Realidad. Argentina RGER.: Amorrortu.

Bourdieu, P., (1997). Razones Prácticas. Sobre la Teoría de la Acción: Editorial Anagrama S. A.

Bourdieu, P. y Wacquant, L., (2005). Una Invitación a la Sociología Reflexiva. Argentina: Siglo XXI Editores.

Castells, M., (1999). La era de la Información. La Sociedad Red Tomo I. Madrid: Siglo XXI Editores. 
Charry, A. Rojas, N., (Compiladores). (2013). La era de los individuos. Actores, política y teoría en la sociedad actual. LOM ediciones.

Giddens, A., (1993). Consecuencias de la Modernidad. Madrid: Alianza Editorial.

Habermas, J., (1987). Teoría de la Acción Comunicativa. Racionalidad de acción y racionalización social. Madrid. Ediciones Taurus.

Heidegger, M. (1962). Ser y Tiempo. Edición digital de: http//www.philosophia.cl

Martuccelli, D. y De Singly, F. (2002). Las Sociologías del Individuo. Santiago, Chile: LOM Ediciones.

Ritzer, G., (2001). Teoría Sociológica Moderna. Mc Graw Hill Interamericana de España. S.A.U.

Schutz, A. y Luckmann Th., (1973). Las Estructuras del mundo de la Vida. Buenos Aires: Amorrortu editores.

Touraine, A., (2001). ¿Podemos vivir juntos? México: Fondo de Cultura Económica.

Weber, M. (2002). Economía y sociedad. Esbozo de sociología comprensiva. Madrid: Fondo de Cultura. 